\title{
Special section on network sciences and engineering
}

The technical committee on "Complex Communication Sciences (CCS)" was launched in April, 2011 as a temporal technical committee of the NOLTA sub-society of IEICE. The main purpose of the CCS activities is to establish a new research field, which can discuss communication and network systems from scientific viewpoints. Since April 2011, the CCS has organized many workshops. Especially, the organizations of "the Korea-Japan joint workshop on CCS" (KJCCS) are major activities of the CCS, which were held three times every year since 2012. Through the KJCCS, good friendships between Korea and Japan are established. Additionally, the special section on Communication Sciences and Engineering of NOLTA, IEICE was issued in April 2014. Through these activities, the CCS was promoted to be a permanent technical committee of NOLTA society of IEICE from April 1st in 2015, which is the same date as this special-section publication!

This special section is organized for transmitting the latest research results related with network and communication engineering from scientific viewpoints. Such topics have been recently presented and discussed at the conferences organized by CCS, especially KJCCS 2013, which was held at Okinawa, Japan in October 2013. This special section includes 15 papers including 2 invited papers, which were accepted through the solemn review processes. The contents of the invited papers are strongly related with the invited talks at KJCCS 2013. The first invited author is a Masanobu Inubushi group from NTT Communication Science Laboratories, NTT Corporation, JAPAN and the second invited one is a Won-Joo Hwang group from Inje University, South Korea.

The guest co-editors would like to express their sincerely thanks to all the authors who submitted their manuscripts to this special section. They also thank to all the editorial committee members and anonymous reviewers. Finally, the guest co-editors would like to state special thanks to the secretaries of the special section, Prof. Hiraku Okada and Prof. Shintaro Arai, for their hard works on publishing this special section.

\section{Dong-Kyue Kim and Hiroo Sekiya \\ Guest Co-Editors}

\section{Editorial Committee of the Special Section}

\section{Guest Co-Editor:}

Dong-Kyue Kim (Hanyang University)

Hiroo Sekiya (Chiba University)

\section{Guest Associate Editors:}

Masashi Aono (Tokyo Institute of Technology)

Mikio Hasegawa (Tokyo University of Science)

Won-Joo Hwang (Inje University)

Song-Ju Kim (NIMS)

Nobuyoshi Komuro (Chiba University)

Hiroaki Kurokawa (Tokyo University of Technology)

Mun-Kyu Lee (Inha University)

Jinhu Lu (RMIT University)

Yasuhiro Tsubo (Ritsumeikan University)

\section{Editors of NOLTA, IEICE:}

Ljiljana Trajkovic (Simon Fraser University)

Michael Tse (Hong Kong Polytechnics University) 


\section{Associate Editors of NOLTA, IEICE:}

Sergio Callegari (University of Bologna)

Francis Lau (Hong Kong Polytechnic University)

Hiroya Nakao (Tokyo Institute of Technology)

\section{Secretaries of the Special Section:}

Shintaro Arai (National Institute of Technology, Kagawa College)

Hiraku Okada (Nagoyoa University) 